\title{
Performance Evaluation of Scheduling Algorithm for Wimax Networks
}

\author{
Radhia Khdhir, Kais Mnif, and Lotfi Kamoun
}

\begin{abstract}
This paper proposes a new scheduling algorithm for IEEE802.16 Broadband wireless Metropolitan Area Networks in TDD mode. The proposed algorithm focuses on an efficient mechanism to serve high priority traffic in congested networks. A detailed simulation study is carried out for the proposed scheduling algorithm and its performance has been compared with some known algorithms such as Proportional Fairness (PF), Adaptive Proportional Fairness (APF) and Round Robin (RR). For performance evaluation and comparison with existing algorithms, we used OPNET 14.5 modeler simulator. The results show that the proposed algorithm is capable of dealing with different users' requirements under congestion conditions.
\end{abstract}

Index Terms-QoS, OFDMA, scheduling algorithms, proportional fairness, wimax.

\section{INTRODUCTION}

The Wimax technology which is based on the IEEE 802.16 standard has a very rich set of features. It is a very promising Broadband Wireless Access (BWA) technology. The objective is to have a highly efficient use of radio resources while serving types of services. However, these services can have different constraints such as maximum latency, tolerated jitter and traffic rate.

The IEEE 802.16 BWA system has three possible physical layers (PHY) as it is shown in Figure 1: Single Carrier (SC), Orthogonal Frequency Division Multiplexing (OFDM), and Orthogonal Frequency Division Multiple Access (OFDMA) [1], [2]. The OFDMA physical layer is the most efficient and most complex one [10]. In OFDMA each subscriber station (SS) can receive some portions of the allocation for the combination of frequency and time so that the channel capacity is efficiently used.

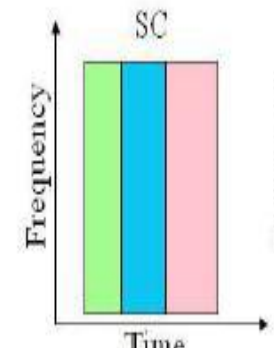

Time

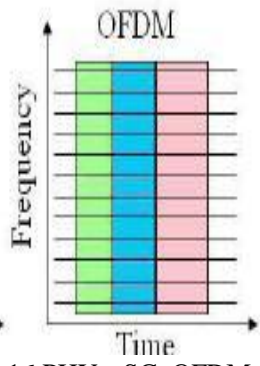

Time

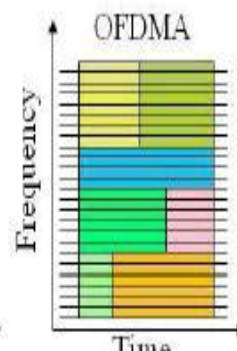

Time
Fig. 1. IEEE 802.16 PHYs: SC, OFDM and OFDMA [5].

Manuscript received October 29, 2012; revised December 24, 2012.

Radhia Khdhir and Lotfi Kamoun are with the LETI laboratory, University of Sfax, Tunisia and National Engineering School of Sfax (ENIS) (e-mail: radiarourou@gmail.com).

Kais Mnif is the Higher Institute of Electronic and Communication of Sfax (ISECS).
Wimax retains only OFDM and OFDMA. For topologies, mesh and Point-to-Multipoint (PMP) modes can be used. Both Duplexing modes are possible: Frequency Division Duplex (FDD) and Time Division Duplex (TDD).

The IEEE 802.16 standard Meduim Access Control (MAC) defines five types of QoS classes: Unsolicited Grant Services (UGS), extended real-time Polling Service (ertPS), real-time Polling Services (rtPS), non extended real -time Polling Services (nrTPS), and Best Effort (BE). UGS is designed to support real time data streams consisting of fixed-size data packets issued at periodic intervals. ertPS is based on the efficiency of both UGS and rtPS. rtPS supports real-time service consisting of variable bit-rate (VBR) data packets that are issued at periodic intervals. nrtPS supports delay tolerant data streams consisting of variable size data packets for which a minimum data rate is required. Best Effort (BE) is used for best effort traffic which does not guarantee any Quality of service (QoS), like the short length FTP or the email. Those service classes are defined in order to satisfy various types of Quality of Service (QoS) requirements.

The MAC (Media Access Control) layer of IEEE802.16 is responsible for allocating resources for SSs (subscriber stations) and active flows is called the scheduling process.

Unlike other parts of IEEE 802.16, Wimax does not specify the scheduling algorithm to be used. Some works have been published about scheduling algorithms for Wimax but the optimal scheduling algorithm is still in open research area [4], [5] and [7]. In this paper, a new scheduling algorithm is proposed to provide a better allocation of resources to different SSs (subscriber stations) based on their priority and QoS parameters.

The remainder of this paper is organized as follows: Section II gives an overview of the scheduling algorithms and problem formulation. In Section III, a detailed description of the proposed algorithm is given. Section IV presents the setup of simulation environment and the simulation results of the algorithm compared to other algorithms. Finally, concluding remarks and directions for future work are given in Section V.

\section{WIMAX SCHEDULING AlgORITHMS}

\section{A. Related Work}

Scheduling algorithms can be classified into two main categories: the first category is based on the channel-unaware schedulers while the second category is based on the channel aware schedulers [5]. Channel aware schedulers don't use any instant information of the channel state condition in making the scheduling decision. The design of those schedulers varies based on the ultimate goal of the scheduler such as fair 
allocation of resources between different SSs or maximizing throughput. However, no single queue algorithm can handle all QoS constrains (jitter, throughput rate, delay...) simultaneously. Indeed, no published researches show how to handle jitter over IEEE802.16 and most researches focus on delay or throughput rate.

Generally, the QoS classes are served in the following order: UGS, ertPS, rtPS, nrtPS and BE. Or on the bases of flow priority, where highest priority flow is served first regardless of its class.

However, even within the same class, there are many constrains that can't be handled through one scheduler[5].for example, UGS class is designed to support real time data streams consisting of fixed-size data packets issued at periodic intervals, maximum latency and priority. Most existing scheduling algorithms give precedence to flows based on one or two of those parameters. For instance Round Robin (RR) distributes resources equally over different queues regardless of flow priority and of its maximum latency.

There is an extension of the RR scheduler, called the Modified Deficit Round Robin (MDRR) scheduler which focuses on giving priority to the lowest data rate flow regardless of its priority [9]. Other scheduling algorithm, like Adaptive Proportional Fairness (APF) which is designed to extend the PF scheduling algorithm provides a satisfaction for the various QoS requirements. The proposed APF tries to differentiate the delay performance of each queue based on the Grant per Type-of- Service (GPTS) principle.

To sum up, the primary objective of a scheduling algorithm is to be able to share the total system bandwidth fairly. Wimax scheduler aims to achieve the optimal usage of resources, to ensure the QoS, to maximize good put and to minimize power consumption while ensuring feasible algorithm complexity and system scalability. However, it is very difficult for any scheduler to handle all the parameters in one step. In this paper, a new scheduling algorithm is proposed to guarantee the maximum scheduling criteria.

\section{B. Problem Formulation}

This research aims to design a scheduling algorithm which is capable of considering all the QoS constraints of all sessions. This algorithm should preserve the main characteristics of each class and be able to discriminate the throughput of each session based on its importance and priority as well. This research focuses on handling UGS, ertPS, rtPS, nrtPS and BE classes. UGS and ertPS classes are very sensitive to delay/bandwidth variation and will be handled via strict priority, where their needs are served before any other class.

\section{Proposed Algorithm}

Adaptive Proportional Fairness (APF) is a scheduling algorithm which aims at extending the PF [7] scheduling to the real time service and provides various QoS requirements. The scheduling scheme is based on the Grant Per Type-of-Service (GPTS) principle, which aims at differentiating the delay performance of each queue. A novel priority function is devised for all the QoS classes, including
UGS, rtPS, nrtPS and ertPS: the Base Station (BS) allocate sing time slots on the queues with the highest priority value. In this research, we aim to propose a scheduling algorithm, called Enhanced Adaptive Proportional Fairness (EAPF) scheduling to extend the APF scheduling to provide various QoS requirements and real time services.

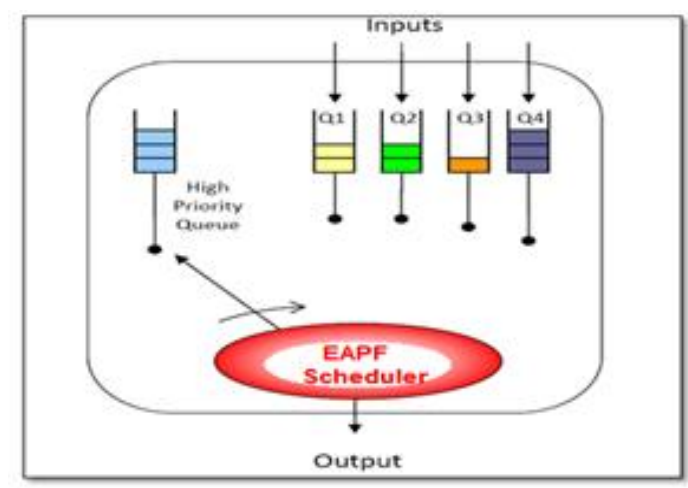

Fig. 2. EAPF algorithm.

The criteria for user satisfaction such as throughput, fairness, etc. do not really reflect the degree of QoS satisfaction. In fact, allocating the same throughput to all users is not always sufficient to ensure an adequate QoS. Let's take the example of two users: one with a low demand while the other with a high demand, the fact of allocating them the same flow will give a great satisfaction for the first and a lower satisfaction for the second. Thus, the aimed fairness is achieved when all users are equally satisfied. In this paper, we suppose that the highest level of fairness is achieved when $\frac{Q_{o} S_{\text {achieved }}}{Q_{o} S_{\text {requied }}}$ is the same for all users. In reality, this is very difficult to achieve. However, the values should at least be close. Thus, we can compare users' satisfaction not through relying on throughput fairness, such as is the case with the APF algorithm which considers that the indicator of satisfaction is $\mathrm{Ri}(\mathrm{t}) \mathrm{ki}(\mathrm{t}) \mathrm{Mi}$, but through relying on the values of $\frac{Q_{o} S_{\text {achieved }}}{Q_{o} S_{\text {requied }}}$. Here, the problem lies in how to measure QoS? Therefore, we have to define a new measurement tool.

Before explaining the functioning of the algorithm, we must first explain the parameter used in our scheduling algorithm. This parameter is studied by researcher Cédric Gueguen in papers [8] and [11]: The physical layer operates using a structure which ensures a good compatibility with the OFDM based transmission mode of the IEEE 802.16. The resource allocation is considered in a centralized approach and performed on a frame by frame basis for the set of mobiles located in the coverage zone of an Base Station (BS). A crucial objective for modern multiple access schemes is to fully support multimedia transmission services. Regarding delay requirements, the meaningful constraint is a limitation of the occurrences of large delay values. We define the concept of delay outage by analogy with the concept of outage used in system coverage planning. A mobile transmission is in delay outage when its packets experienced a delay greater than a given threshold. The Packet Delay Outage Ratio (PDOR) target is defined as the maximum ratio of packets that 
may be delivered after this fixed delay threshold. In the following, Tk represents the delay threshold of the mobile $\mathrm{k}$ as is shown in Figure 3. The PDOR experienced by each transmission is tracked all along their lifetime. At each transmission of a packet of mobile $\mathrm{k}$, the total number of packets whose delay exceeded the threshold divided by the total number of packets transmitted since the beginning of the connection is computed. The result is denoted PDORk.

In this manner, we can say whether a user ensures their satisfaction or not. A very important remark is that the satisfaction of a user is in inverse proportion to its PDOR. In fact, if the rate of packets which arrive late is high, the level of satisfaction of the user is low, while if PDOR is low, we can say that the level of satisfaction is considered high. Besides, if PDOR exceeds PDOR target, then we can consider that the user is unsatisfied.

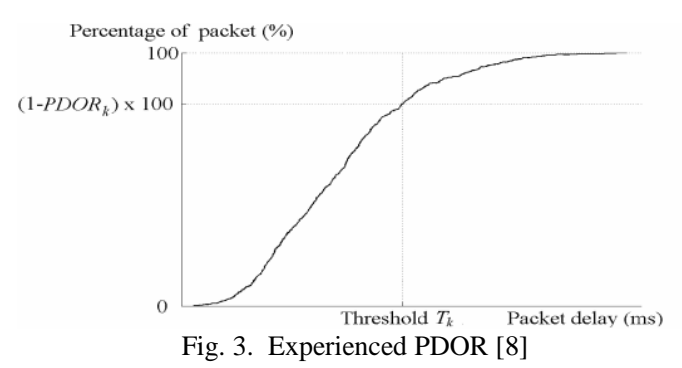

As we have already mentioned, the APF scheduler (Adaptive Proportional fairness) is an algorithm that takes into account the different requirements of quality of services. Moreover, packets coming from various SSs will be classified into five queues: one for UGS, one for BE, then one for ertPS, one for nrtPS and eventually one for rtPS. In order to serve a queue, the scheduler must determine the queue with the highest priority according to the following priority function:

$$
\frac{W_{i}(t) \times K_{i}(t) \times M_{i}(t)}{R_{i}(t)}
$$

where:

- $i$ : between [1..5],

- $W_{i}(t)$ : the transmission capacity at time $t$,

- $K_{\mathrm{i}}(\mathrm{t})$ :the number of communication of the $i^{\text {th }}$ queues,

- $M_{\mathrm{i}}(\mathrm{t})$ : the minimum rate requirement,

- $R_{i}(\mathrm{t})$ : the estimated average:

Although the APF is ranked among the "intelligent" schedulers (as it takes into consideration the instant variations of the channel) and seeks to maximize the throughput, this algorithm achieves a relative fairness between users as it gives favor to serve SSs which are close to BS. In fact, APF does not really deal with the positions of users in the cell. In fact, if we assess the APF algorithm with the new measurement parameter (PDORk), we find that a user who is far away from the BS does not have the same value of $\frac{Q_{o} S_{\text {achieved }}}{Q_{o} S_{\text {requied }}}$ compared to a closer user. Thus, users do not have the same degree of satisfaction.

The fact of taking into consideration only the parameters of equation (1) in the scheduling process enables us to reach a system overall throughput close to the optimal. However, aiming at increasing the throughputs only does not guarantee good QoS. In this context, it is therefore necessary to provide fairness and service differentiation, which cannot be achieved without the addition of another decision criterion in the allocation of priority. In addition to (1), we introduce a second component $\left(1+10^{6} \times P^{2} O R_{k}^{3}\right)$ that have been studied and tested on [8] and [11]. Thus, our EAPF algorithm (Enhanced Adaptive Fairness Proportional) does not seek only to maximize the throughput for a better connection, but it also seeks to achieve fairness among the different users. The proposed priority function is as follows:

$$
Y_{i}=\frac{W_{i}(t) \times K_{i}(t) \times M_{i}(t)}{R_{i}(t)}+\left(1+10^{6} \times P D O R_{k}^{3}\right)
$$

The conditions and initialization for our algorithm are the following:

- $P D O R_{i}=\min P D O R_{\text {flux }}$ : It is the rate of packets coming after Tk threshold in a queue.

- $P D O R_{\text {target }(i)}=\min P D O R_{\text {target }}$ flux: the maximum rate (the rate from which the application will be disturbed and the user will be considered unsatisfied) of late packets in a queue equals the minimum rate of late packets per flow belonging to this queue.

- $P D O R_{i}<P D O R_{\text {target(i) }}$ : rate of packets coming after $\mathrm{T}_{\mathrm{k}}$ threshold in a queue must be less than the maximum rate of late packets in a queue.

The algorithm will serve the five queues according to a priority function described by equation (2) and the course of steps as shown in Figure 4.

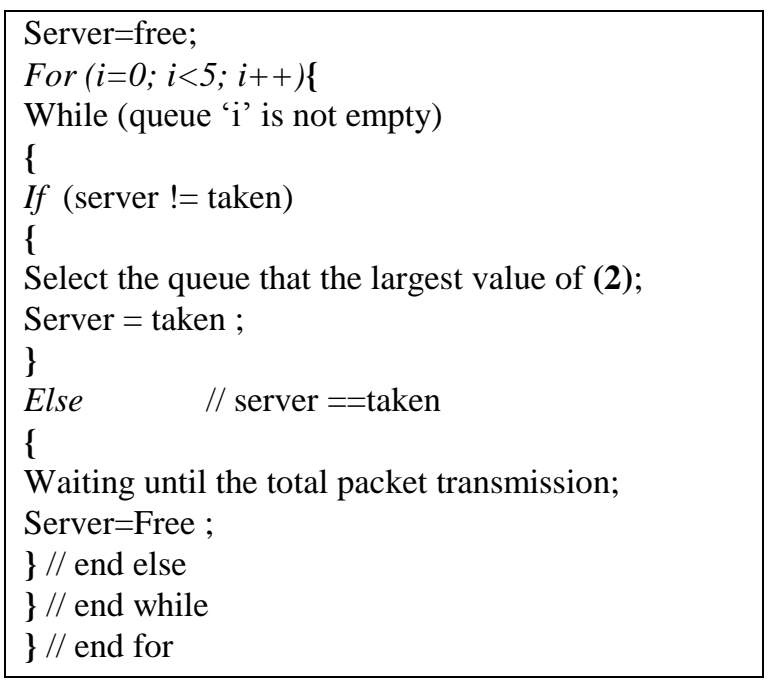

Fig. 4. Flowchart of the algorithm (EAPF)

\section{Simulation Model AND RESUlts}

\section{A. Simulation Model}

The overall objective of the simulation model is to analyze the behavior and performance of the proposed algorithm in a congested uplink domain. The simulations have been performed using Opnet Modeler, version 14.5 [13]. The important parameters used to configure the PHY and MAC 
layers are summarized in Table I.

TABLE I: Main PARAmetres Of The Simulation Model

\begin{tabular}{ll}
\hline Parameter & Value \\
\hline Model & PMP \\
\hline Wimax channel bandwidth & $10 \mathrm{Mhz}$ \\
\hline Frame duration & $5 \mathrm{~ms}$ \\
\hline Symbol duration & $12.6 \mu \mathrm{s}$ \\
\hline Number of symbol per PS & 4 \\
\hline Initial modulation & $1 / 2$ QPSK \\
\hline W (bit/symbol) & 1 \\
\hline
\end{tabular}

The simulation environment consists of one BS and $23 \mathrm{SSs}$ operating in IEEE 802.16 PMP mode. There will be one service flow between each SS and the BS.

For all SSs, we chose a modulation $1 / 2$ QPSK and each SS will establish one or more sessions to each class of service.

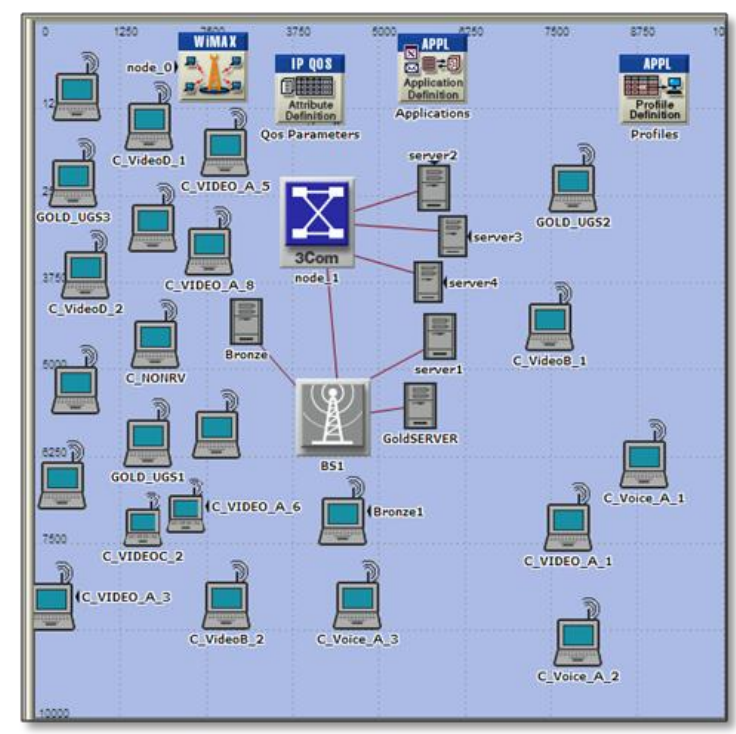

Fig. 5. System model implementation in Opnet

\section{B. Results}

In this section, the output of simulation is shown and analyzed: we compared the proposed Enhanced Adaptive Proportional Fairness (EAPF) scheduling with the APF [7] and MDRR [9].

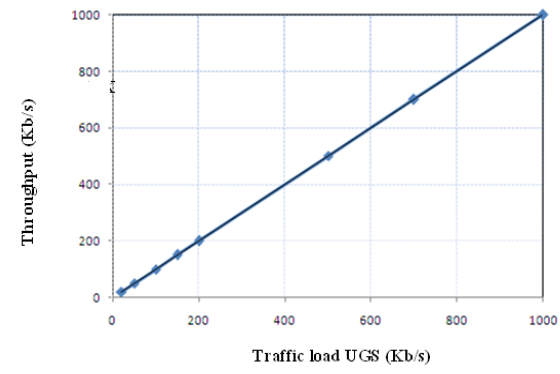

Fig. 6. Throughput according to traffic load

Figure 6 shows a linear curve which represents the throughput of UGS connections according to the traffic load. The load present in the network increases proportionately with the connections traffic because all UGS subscribers should be served as soon as there is a request for SS. In other words, the SS which requests UGS class must always be the first satisfied.

Figures 7 and 8 compare the variations rate for the far and close subscribers to the BS with the same MDRR, APF and EAPF. The observation of another parameter, which is the average occupancy rate of queues, helps us to better understand the performance of the proposed algorithm. In fact, the EAPF algorithm is based on PDOR parameter (the rate of packets coming after $\mathrm{Tk}$ threshold) to better serve the subscribers and in particular the remote SSs. In fact, when a user moves away from the Base Station, his throughput as well as his QoS are reduced, and this leads to the increase of his PDORk.

Since EAPF takes into consideration this parameter, the user will have a more important priority that allows a return to normal and therefore to better manage the queues and thus minimize saturation. In fact, concerning SSs away from the Base Station, the EAPF algorithm increases the proportion of instant processing of packets. This is clearly expressed in Figure 7. In fact, the EAPF algorithm limits the excessive accumulation of packets in the queues and it remains always more efficient than APF as it obliges the unprocessed packet rate to remain below PDORtarget values. With the MDRR scheduler, a relatively light increase of the traffic load (around $600 \mathrm{~kb} / \mathrm{s}$ ) leads to the accumulation of unprocessed packets, thus to the saturation of the queues.

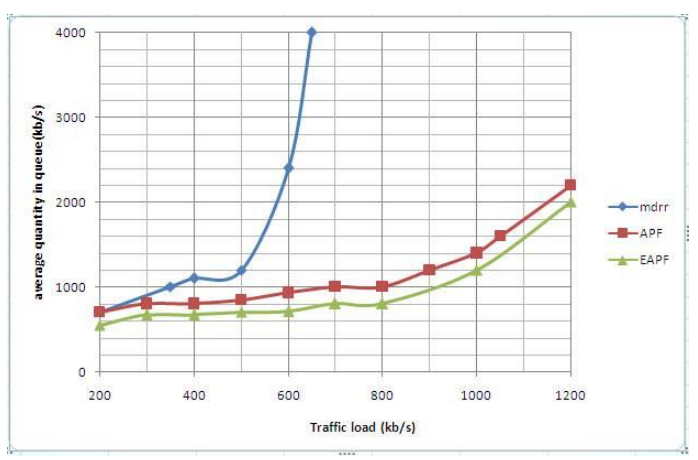

Fig. 7. Average quantity in queues $(\mathrm{Kb} / \mathrm{s})$ of remotes SSs

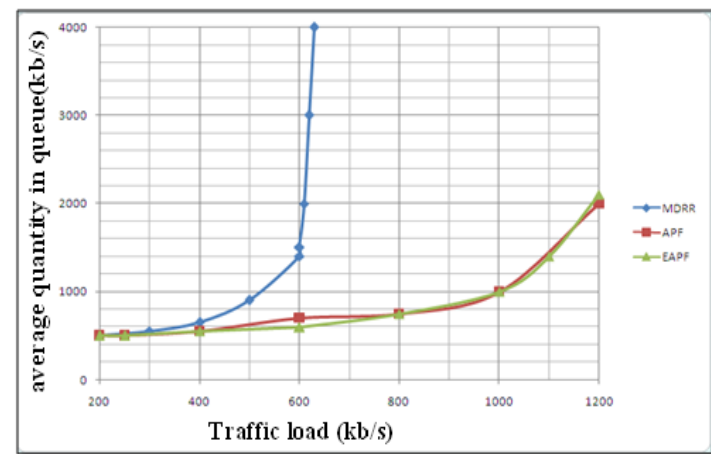

Fig. 8. Average quantity in queues $(\mathrm{Kb} / \mathrm{s})$ of closes SSs

Figure 8 shows the occupancy of queues for mobiles which are close to the BS. Concerning subscribers away from the BS, the queues with MDRR algorithm become saturated as soon as we increase the traffic load. We note that the occupation of queues with APF and EAPF algorithms is almost the same for the mobiles close to the BS. This is because the PDOR value considerably decreases for the mobiles situated in the vicinity of the BS. Thus, the priority functions of both algorithms EAPF and APF become closer than those of mobiles remote 
from the BS.

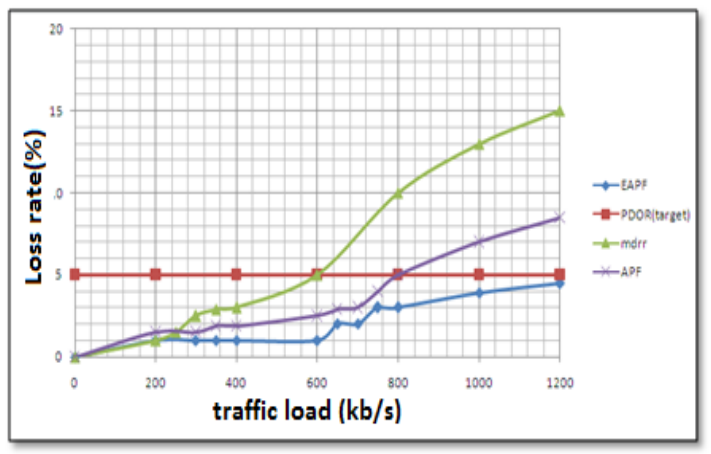

Fig. 9. Packets loss rate

The rate of packet loss shown in Figure 9: the red line represents the value of PDORtarget (the maximum rate of packets coming after the Tk threshold not to be exceeded), the green line represents the MDRR scheduler, the purple line is for the APF and eventually the blue curve represents the result of the proposed solution.

We notice that the packets loss caused by our solution, that is to say EAPF, is less than those given by MDRR and APF. As we have already seen, the EAPF algorithm offers the opportunity to reduce the size of the queue compared to the two other approaches, which reduces the likelihood of having full data queues.

Therefore, it decreases the probability of packet rejection. The loss rate increases when we apply the MDRR algorithm: we notice that when the traffic load is equal to $600 \mathrm{kbit} / \mathrm{s}$, the rate of loss exceeds the threshold (the PDORtarget). This is caused by the saturation of queues represented by Figure 10. Thus, with the AFP scheduler, the packet rate is relatively high and reaches $5 \%$ as soon as the payload exceeds $800 \mathrm{kbit}$ / s (the threshold to keep the connection undisturbed). To conclude, the EAPF algorithm offers the opportunity to minimize the data waiting time, reduces the size of queues and especially decreases the loss rate of packets. These results are deemed important and beneficial.

For each rtPS user, the BS must allocate a throughput higher than or equal to the reserved minimum throughput. Thus, the notion of throughput for this class is very interesting especially for video stream transfer applications. Figure 10 shows the throughput of rtPS-type connections according to the traffic load. According to the simulation results, we notice that the proposed algorithm ensures a throughput of rtPS connections that reaches $9.5 \mathrm{Mbit} / \mathrm{s}$ and always remains more efficient than the APF and MDRR algorithm.

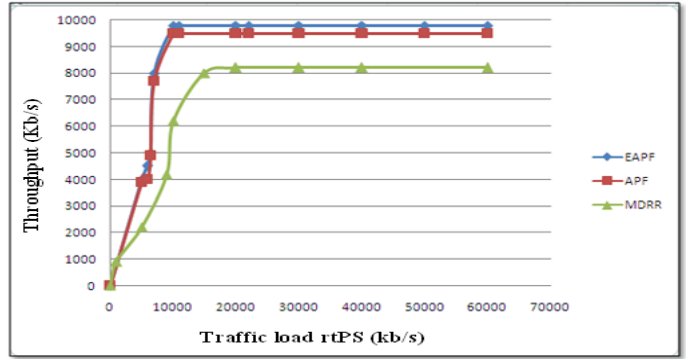

Fig. 10. Throughput as a function of traffic load (class rtPS)

\section{CONCLUSION}

In this paper, a new scheduling algorithm for IEEE 802.16 wireless MAN in PMP is proposed called "Enhanced Adaptive Proportional Fairness". This algorithm proposes a mechanism to enable the BS scheduler to balance between serving high and low priority traffic simultaneously.

\section{REFERENCES}

[1] DRAFT standard for local and metropolitan area networks, IEEE P802.16Rev2/D2, Part 16: Air Interface for Broadband Wireless Access Systems, Dec. 2007.

[2] Working group on broadband wireless access. IEEE 802.16. [Online]. Available: http://wirelessman.org

[3] L. Nuaymi, Wimax technology for brodband wireless access, John Wiley and Sons, 2007.

[4] A. Belghith, "Comparison of WiMAX scheduling algorithms and proposals for the rtPS QoS class," ENST Bretagne.

[5] A. E. Shinnawy, "Two-Step scheduling algorithm for IEEE802.16 wireless networks," 12th Communication Technology (ICCT), 2010.

[6] Opnet. [Online]. Available: http://www.opnet.com

[7] X. J. Meng, "An efficient scheduling for divers QoS requirements in WiMAX”, Electrical and Computer Engineering Waterloo, Ontario, Canada, 2007

[8] C. Gueguen and S. Baey, "Weighted fair opportunistic scheduling for multimedia QoS support in multiuser OFDM wireless networks," IEEE 19th Inernational Symposuim on Personal, Indoor AND Mobile Radio communications, 2008.

[9] M. Shreedhar and G. Varghese, "Efficient fair queuing using deficit round robin," IEEE/ACM Transaction on networking, July 1996.

[10] R. Jain, C. S. In, A. Tamimi, "Schudeling in IEEE 802.16e mobile wimax networks: Key issues and survey," IEEE journal on special Area in communication (JSAC), vol. 27, no. 2, February 2009.

[11] C. Gueguen and S. Baey, "Improving mobile satisfaction in multiuser OFDM wirless networks," Ecole ete RESCOM 15-21 June 2008, Saint-Jean-cap-Ferrat.

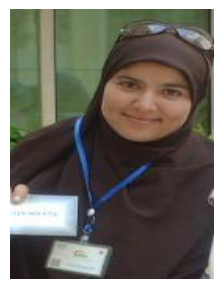

Radhia Khdir was from Tunisia. She was born in karkina Sfax on 1986, A PHD student at the National Engineering School of Sfax (ENIS), University of Sfax (Tunisia). She obtained a Master Degree on Computer Science, Dedicated Computer System New Technologies (NTSID) from ENIS. She is a member of Research Groups (LETI) Laboratory since 2010. Her research activity is in the domain of Radio resource management (Power Control, Scheduling, Admission Control, Congestion Control) in Cellular CDMA and OFDMA networks. She is a lecturer at the faculty of sciences of Gafsa, Tunisia where he teach different courses related to computer science. 